\title{
Exclusive leptonic and radiative $B$ meson decays at Belle
}

\author{
Shohei Nishida ${ }^{* \dagger}$ \\ KEK \\ E-mail: shohei.nishida@kek.jp
}

\begin{abstract}
Measurements of $B \rightarrow K^{(*)} \ell^{+} \ell^{-}$and $B \rightarrow K \eta^{\prime} \gamma$ at Belle are reported. We show the updated measurements of the partial branching fraction, forward-backward asymmetry and isospin asymmetry for $B \rightarrow K^{(*)} \ell^{+} \ell^{-}$. We also find the first evidence of $B^{+} \rightarrow K^{+} \eta^{\prime} \gamma$ with a branching fraction of $(3.6 \pm 1.2 \pm 0.3) \times 10^{-6}$. The results are based on a dataset of 657 million $B \bar{B}$ pairs collected at the Belle detector at the KEKB asymmetric $e^{+} e^{-}$collider.
\end{abstract}

European Physical Society Europhysics Conference on High Energy Physics, EPS-HEP 2009,

July 16 - 222009

Krakow, Poland

*Speaker.

$\dagger$ on behalf of the Belle Collaboration 


\section{Introduction}

The $b \rightarrow s \gamma$ and $b \rightarrow s \ell^{+} \ell^{-}$transitions are flavor-changing neutral current processes, and are sensitive to physics beyond the Standard Model (SM). They proceed through an one-loop electroweak penguin diagram (or box diagram for leptonic decays). Although the branching fractions of the exclusive decays suffer large uncertainty in theoretical calculations, there are theoretically clean observables useful for the search for the New Physics (NP). For example, since photons in radiative $B$ decays are almost polarized, large mixing-induced $C P$ violation is an indication of NP.

The $b \rightarrow s \ell^{+} \ell^{-}$process is sensitive to the effective Wilson coefficients $C_{7}, C_{9}$ and $C_{10}$, which describe the amplitudes from the electromagnetic penguin, the vector electroweak, and the axialvector electroweak contributions, respectively. The inclusive $b \rightarrow s \gamma$ branching fraction makes a constraint for $\left|C_{7}\right|$, but not for $C_{9}, C_{10}$ and the sign of $C_{7}$. Therefore, the $b \rightarrow s \ell^{+} \ell^{-}$process can probe the NP that are not accessible by the radiative decays.

In this proceedings, we report measurements of the $B \rightarrow K^{(*)} \ell^{+} \ell^{-}$[1] and $B \rightarrow K \eta^{\prime} \gamma$ [2] decays at Belle [3]. The results are based on a data sample of 657 million $B \bar{B}$ pairs collected at the Belle detector at the KEKB asymmetric $e^{+} e^{-}$collider [4].

\section{2. $B \rightarrow K^{(*)} \ell^{+} \ell^{-}$}

The analysis of $B \rightarrow K^{(*)} \ell^{+} \ell^{-}$is performed by combining $K$ or $K^{*}$ and two leptons ( $e$ or $\mu$ ) with opposite charge, where $K^{*}$ is reconstructed from $K^{+} \pi^{-}, K_{S}^{0} \pi^{+}$and $K^{+} \pi^{0}$. Here, photons between $20 \mathrm{MeV}$ and $500 \mathrm{MeV}$ within a $50 \mathrm{mrad}$ cone along the direction of electrons are included in the reconstruction in order to recover the Bremsstrahlung photons from electrons.

The dominant backgrounds are the continuum and semi-leptonic $B$ decay events. These backgrounds are suppressed using likelihood ratio formed from event shape variables, missing mass, etc. The contribution from $B \rightarrow J / \psi X$ and $\psi^{\prime} X$ is removed by applying veto for events whose $q^{2}$ $\left(=M_{\ell^{+} \ell^{-}}^{2}\right.$ ) is in the following region (in unit of $\mathrm{GeV}^{2} / c^{2}$ ): $8.11<q^{2}<10.03$ or $12.15<q^{2}<14.11$ for electron pairs and $8.68<q^{2}<10.09$ or $12.86<q^{2}<14.18$ for muon pairs. There exists some peaking background from $B \rightarrow K^{(*)} \pi \pi$ events, where both $\pi$ 's are misidentified as leptons. This contribution is estimated from the data.

Signal yields are extracted by a two-dimensional fit on $M_{\mathrm{bc}}$ and $M_{K \pi}$ for $B \rightarrow K^{*} \ell^{+} \ell^{-}$, while one-dimensional $M_{\mathrm{bc}}$ fit is used for $B \rightarrow K \ell^{+} \ell^{-}$. We find $230 \pm 24$ events for $K^{*} \ell^{+} \ell^{-}$, and $166 \pm 15$ events for $K \ell^{+} \ell^{-}$.

Figure 1a and 1b show the partial branching fractions of $B \rightarrow K^{*} \ell^{+} \ell^{-}$and $B \rightarrow K \ell^{+} \ell^{-}$. The results are consistent with the SM theoretical prediction [5]. The total branching fractions are calculated to be $\left(19.7_{-1.0}^{+1.1} \pm 0.9\right) \times 10^{-7}$ and $\left(4.8_{-0.4}^{+0.5} \pm 0.3\right) \times 10^{-7}$. In this calculation, the lepton flavor ratio $R_{K^{(*)}} \equiv \mathscr{B}\left(B \rightarrow K^{(*)} \mu^{+} \mu^{-}\right) / \mathscr{B}\left(B \rightarrow K^{(*)} e^{+} e^{-}\right)$is assumed to be the values in the SM: $R_{K}=1$ and $R_{K^{*}}=0.75$ (due to the photon pole). This ratio is also sensitive to NP, and takes larger value in the Higgs doublet model at large $\tan \beta$. Our data show $R_{K^{*}}=0.83 \pm 0.17 \pm 0.05$ and $R_{K}=1.03 \pm 0.19 \pm 0.06$, which are consistent with the SM.

For $B \rightarrow K^{*} \ell^{+} \ell^{-}$, the $K^{*}$ longitudinal polarization fraction $F_{L}$ and the forward backward asymmetry $A_{\mathrm{FB}}$ are extracted using the relations $d \Gamma / d \cos \theta_{K^{*}}=\frac{3}{2} F_{L} \cos ^{2} \theta_{K^{*}}+\frac{3}{4}\left(1-F_{L}\right) \sin ^{2} \theta_{K^{*}}$ and $d \Gamma / d \cos \theta_{B \ell}=\frac{3}{4} \sin ^{2} \theta_{B l}+\frac{3}{8}\left(1-F_{L}\right)\left(1+\cos ^{2} \theta_{K^{*}}\right)+A_{\mathrm{FB}} \cos \theta_{B \ell}$, where $\theta_{K^{*}}$ is the angle between 

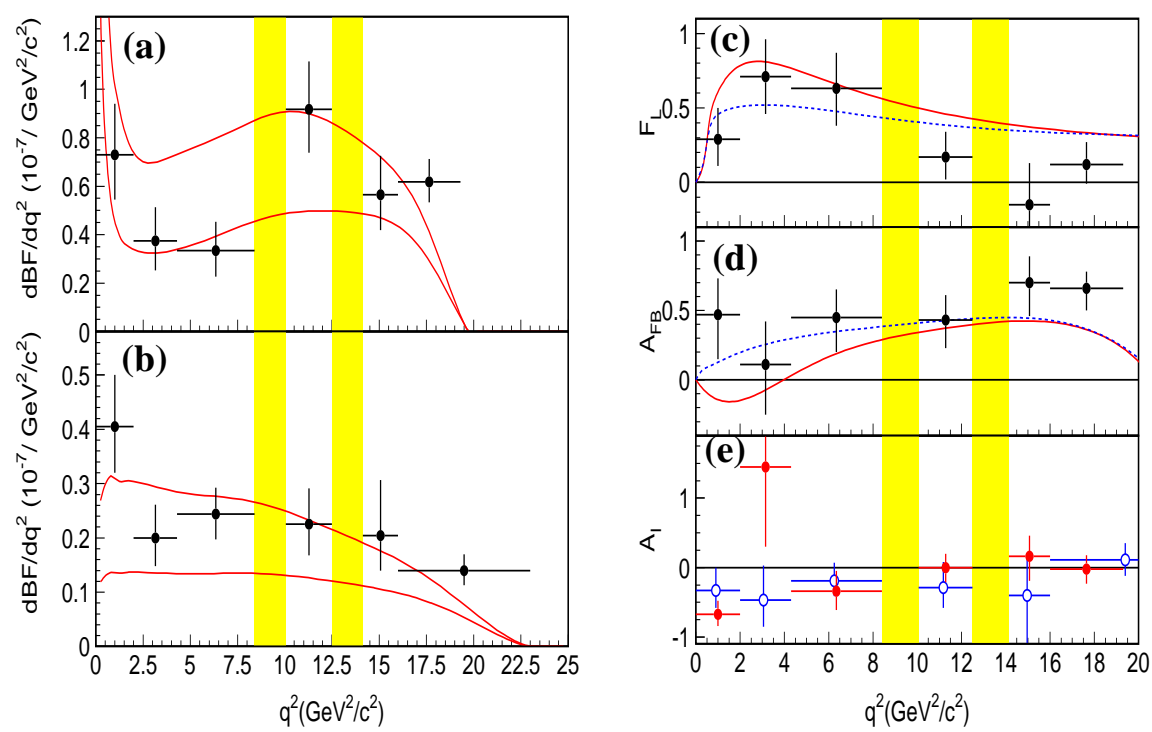

Figure 1: Differential branching fractions for (a) $K^{*} \ell^{+} \ell^{-}$and (b) $K \ell^{+} \ell^{-}$. The two shaded regions are veto windows to reject $J / \psi\left(\psi^{\prime}\right) X$ events. The solid curves show the SM theoretical predictions with the minimum and maximum allowed form factors. (c) and (d) show the fit results for $F_{L}$ and $A_{F B}$ in $K^{*} \ell^{+} \ell^{-}$, together with the solid (dotted) curve representing the $\mathrm{SM}\left(C_{7}=-C_{7}^{S M}\right)$ prediction. (e) is the $A_{I}$ asymmetry for the $K^{*} \ell^{+} \ell^{-}$(closed circles) and $K \ell^{+} \ell^{-}$(open circles) modes. All the plots are shown as a function of $q^{2}$.

$K$ and opposite of $B$ in $K^{*}$ rest frame and $\theta_{B l}$ is the angle between $\ell^{+}\left(\ell^{-}\right)$and opposite of $B(\bar{B})$ in the dilepton rest frame. The $q^{2}$ dependence of $F_{L}$ and $A_{\mathrm{FB}}$ is shown in Fig. 1c and 1d. For reference, the SM prediction and a non-SM scenario with the flipped $C_{7}$ case are plotted. The results are consistent with the SM, although the flipped $C_{7}$ case looks more favored for $A_{\mathrm{FB}}$. Figure 1e shows the measured isospin asymmetry $A_{I}$. BaBar observed large discrepancy from null asymmetry at low $q^{2}$ region [6], but our results are consistent with null asymmetry in all the $q^{2}$ region.

\section{3. $B \rightarrow K \eta^{\prime} \gamma$}

Better understanding of the exclusive final states in radiative $B$ decays reduces the uncertainty in the inclusive measurement of $B \rightarrow X_{s} \gamma$ and $B \rightarrow X_{s} \ell^{+} \ell^{-}$. The decay $B \rightarrow K \eta^{\prime} \gamma$ is one of such exclusive decays, but the previous measurement by BaBar [7] only gives the upper limit of $6.6 \times$ $10^{-6}\left(4.2 \times 10^{-6}\right)$ for the charged (neutral) mode. Comparison of the branching fraction of $B \rightarrow$ $K \eta^{\prime} \gamma$ and $B \rightarrow K \eta \gamma$ provides additional input to the QCD calculation techniques that predict the hierarchy of $B \rightarrow K \eta$ and $B \rightarrow K \eta^{\prime}$ [8]. The neutral mode $B^{0} \rightarrow K_{S}^{0} \eta^{\prime} \gamma$ can also be used to study time-dependent $C P$ asymmetry to search for NP.

The reconstruction of $B^{+} \rightarrow K^{+} \eta^{\prime} \gamma$ and $B^{0} \rightarrow K_{S}^{0} \eta^{\prime} \gamma$ is performed via $\eta^{\prime} \rightarrow \eta \pi^{+} \pi^{-}$and $\eta^{\prime} \rightarrow \rho \gamma$, where $\eta$ is reconstructed from $\gamma \gamma$ and $\pi^{+} \pi^{-} \pi^{0}$. The invariant mass of $K \eta^{\prime}$ system is required to be less than $3.4 \mathrm{GeV} / c^{2}$. The dominant background from the continuum process is suppressed by the likelihood ratio from event shape variables and $B$ flight direction. Background from the $b \rightarrow c$ process is also suppressed by vetoing $D^{0}$; for charged mode, an event is rejected if the invariant mass of the kaon used in the reconstruction and any pion with charge opposite to 
the kaon satisfies $1.84<M_{K^{-} \pi^{+}}<1.89 \mathrm{GeV} / c^{2}$. The contamination from $B \rightarrow J / \psi K \rightarrow \eta^{\prime} \gamma K$ is negligible after rejecting events with $3.07<M_{\eta^{\prime} \gamma}<3.12 \mathrm{GeV} / c^{2}$, where $M_{\eta^{\prime} \gamma}$ is the invariant mass of $\eta^{\prime} \gamma$.

The signal is extracted by performing a 2-dimensional fit on $M_{\mathrm{bc}}$ and $\Delta E$. Figure 2 shows the fit result. For $B^{+} \rightarrow K^{+} \eta^{\prime} \gamma$, we find $33_{-11}^{+12}$ events with a statistical significance (including systematic errors) of $3.3 \sigma$. This is the first evidence of the $B^{+} \rightarrow K^{+} \eta^{\prime} \gamma$ decay. The branching fraction is measured to be $(3.6 \pm 1.2 \pm 0.3) \times 10^{-6}$. The signal yield for $B^{0} \rightarrow K_{S}^{0} \eta^{\prime} \gamma$ is $5.0_{-4.0}^{+5.0}$, and the branching fraction is measured to be $\left(2.5_{-1.9}^{+2.4}+0.4\right) \times 10^{-6}$. Since the significance is only $1.3 \sigma$, we set the upper limit of $6.4 \times 10^{-6}$ for the neutral mode.

(a) $M_{\mathrm{bc}}$ for $K^{+} \eta^{\prime} \gamma$

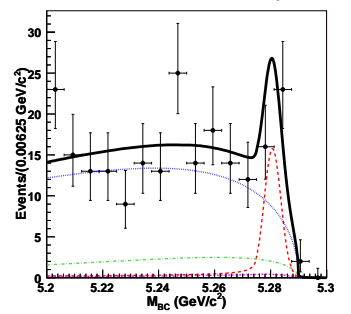

(b) $\Delta E$ for $K^{+} \eta^{\prime} \gamma$

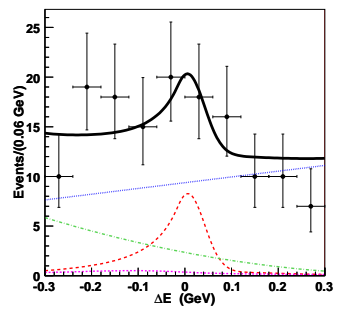

(c) $M_{\mathrm{bc}}$ for $K_{S}^{0} \eta^{\prime} \gamma$

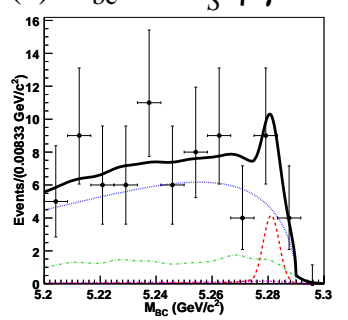

(d) $\Delta E$ for $K_{S}^{0} \eta^{\prime} \gamma$

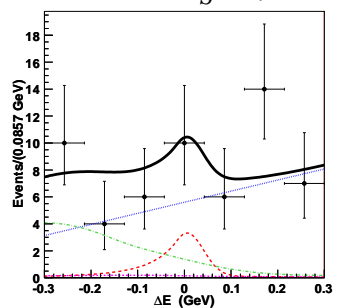

Figure 2: $M_{\mathrm{bc}}$ and $\Delta E$ distributions for $B^{+} \rightarrow K^{+} \eta^{\prime} \gamma$ and $B^{0} \rightarrow K_{S}^{0} \eta^{\prime} \gamma$. Projections to $M_{\mathrm{bc}}(\Delta E)$ signal region are plotted in $\Delta E\left(M_{\mathrm{bc}}\right)$ plots. Points with error bars are the data, while the solid lines are fit results. The dotted, dot-dashed, long dashed and short dashed lines represent the continuum, $b \rightarrow c$, signal, $b \rightarrow u$ components, respectively.

\section{Conclusion}

As exclusive decay modes of the $b \rightarrow s \gamma$ and $b \rightarrow s \ell^{+} \ell^{-}$process, $B \rightarrow K^{(*)} \ell^{+} \ell^{-}$and $B \rightarrow K \eta^{\prime} \gamma$ are studied in Belle. For $B \rightarrow K \eta^{\prime} \gamma \gamma$, we have found the first evidence in the charged mode. For $B \rightarrow K^{(*)} \ell^{+} \ell^{-}$, we have reported the updated results on the branching fractions and the forwardbackward asymmetry. So far, all the results are consistent with the SM, and more luminosity is necessary for precise test of the SM.

\section{References}

[1] J.-T. Wei et al. (Belle Collaboration), arXiv:0904.0770 [hep-ex].

[2] R. Wedd et al. (Belle Collaboration), arXiv:0810.0804 [hep-ex].

[3] A. Abashian et al., Nucl. Instr. and Meth. A479 (2002) 117.

[4] S. Kurokawa and E. Kikutani, Nucl. Instrum. Meth., A499, 1 (2003), and other papers included in this Volume.

[5] A. Ali et al., Phys. Rev. D61, 074024 (2000); A. Ali et al., Phys. Rev. D66, 034002 (2002).

[6] B. Aubert et al. (BaBar Collaboration), Phys. Rev. Lett. 102, 091803 (2009).

[7] B. Aubert et al. (BaBar Collaboration), Phys. Rev., D74, 031102(R) (2006).

[8] M. Beneke and M. Neubert, Nucl. Phys. B651, 225 (2003). 